\title{
On determining defects identity in carbon nanotubes using charge probes
}

\author{
T. Kostyrko ${ }^{\mathrm{a}}$, V. M. García-Suárez ${ }^{\mathrm{b}, \mathrm{c}}$, M. Wawrzyniak-Adamczewska ${ }^{\mathrm{a}}$, J. Ferrer ${ }^{\mathrm{b}, \mathrm{c}}$ \\ ${ }^{a}$ Faculty of Physics, A. Mickiewicz University, ul. Umultowska 85, 61-614 Poznań, Poland \\ ${ }^{b}$ Departamento de Física, Universidad de Oviedo, 33007 Oviedo, Spain \\ ${ }^{c}$ Nanomaterials and Nanotechnology Research Center (CINN), Oviedo, Spain
}

\begin{abstract}
A metallic carbon nanotube with point-like defects under influence of a local potential due to a point charge probe is theoretically studied. A combination of density functional theory and the Landauer-Büttiker formalism is used to compute the electronic conductance in the zero-voltage limit. From a collection of the results obtained by varying the probe position around different defects the conductance maps are created. The analysis of the conductance maps allows us to formulate conditions under which several point-like defects (the Stone-Wales defect, a simple carbon vacancy, hydrogen-passivated vacancies) can be distinguished and identified in experiments with the help of scanning probe microscopy.
\end{abstract}

Keywords: carbon nanotubes, electronic conductance, defects, scanning probe microscopy, density functional theory

\section{Introduction}

Detection and characterization of defect states in carbon nanotubes (CNTs) still make an important challenge despite many years of research (see references [1-3]). Understanding the role of defects in the transport properties[4-6] of these one dimensional systems is a matter of special importance. CNTs in the field of nanoelectronics have already been used in many devices[7, 8], which include wires, field-effect transistors, memory devices and biochemical sensors. An essential aspect of this technology is that the electronic structure and location of a single defect may decide the functional properties of a device.

The state-of-art experimental methods of detecting, locating and identifying defects use chemical reactivity of defect sites[9], direct imaging by means of high-resolution transmission electron microscopy (HR-TEM) or different variants of scanning probe microscopy (SPM)[2]. The scanning gate microscopy (SGM) belongs to the latter group. The method provides information directly relevant for analysis of transport phenomena, by mapping the electrical conductance of a device as a function of the position of a tip and its electrostatic potential[10-15].

So far a successful identification of various defects in CNTs with the above techniques remains a challenge. One of the reasons is the low density of simple intrinsic point defects[2], like a single vacancy or a Stone-Wales[16] (or 5775) defect. This is due to their high energy of creation[17] and consequently a small thermodynamic probability of formation during synthesis of CNTs. The number of defects can be increased using irradiation[18, 3], heat treatment[19], laser pulses[20] or sonication[21]. An example of simple point defect that were created in CNTs in this way is the 5775 defect, resulting from 90 degree rotation of the $\mathrm{C}-\mathrm{C}$ bond and consisting of two heptagon-pentagon pairs, subsequently identified by means of HR-TEM technique[19]. Similar methods were recently used to create monovacancies in a single graphitic layer. Depending on the ambient condition the vacancies were found in bare or in the hydrogen-passivated forms, as was confirmed by scanning tunneling microscope studies[22].

On the theoretical side, information required to identify defects in experiments is not yet complete. A large number of computations done so far concerned the role of various defects in the transport properties in zero external electric field[1,6]. Moreover, a number of papers studied the effect of a uniform electric field on the electronic structure[23] 
and the transport properties[24-26] of CNTs with defects. There was also a first principle study of perfect CNT under the influence of spatially variable external electric fields[27]. However, none of the previous first principles computations simulates a typical SGM measurement in a CNT with defects, where the conductance response to a variable local gate position is recorded.

The purpose of the present paper is to provide new computational results that can be compared more directly with SPM experiments and propose new schemes that allow for the identification of defects by using local probes. Unlike previous works, we investigate the transport properties of a singlewall CNT with a defect in an inhomogeneous electric field. We use density functional theory (DFT) and quantum transport simulations that allow us to study rather big systems. We employ as source of the electric field a point charge, which provides an electrostatic potential that is an idealized representation of the one created by a scanning gate. By varying the position of the probe we compute a spatial (2-dimensional) conductance map that can be used in interpreting the experiments.

In Sec.II we provide the details of our model and the computational details. In Sec.III we discuss the numerical results and in Sec.III we provide our general conclusions.

\section{Details of computations}

We considered a single-wall armchair $(10,10)$ CNT, which exhibits a metallic electronic structure. It has a diameter of $1.4 \mathrm{~nm}$, typical to CNTs obtained in synthesis[28]. The nanotube includes in the central part of the model of a two-terminal device a single intrinsic point defect: a vacancy or a Stone-Wales (5775) defect. The point-charge probe (PCP) has a positive charge that can be either $Q=+|e|$ or $Q=+2|e|$ and is located about 3.5 to $4.5 \AA$ above the CNT surface (see figure 1 ). The position of the PCP is varied in a series of computations along a rectangular region of size: $0.86 \times 0.99 \mathrm{~nm}^{2}$ that is centered near the defect. We included 13 unit cells of the CNT in central part of the device, which ensures a smooth transition between the central part (scattering region) and the leads. This guarantees that the reflection of electrons incident at the CNT-lead boundaries is small. The effect of details of binding between the nanotube and the metallic electrodes is also minimized



Figure 1: (color online) The CNT junction with the highlighted atoms of 5775 defect. The point charge is indicated with a red ball, and atoms included in the leads are colored in blue[29].

by using perfect semi-infinite $(10,10)$ nanotubes as leads.

In this work we used the SIESTA code[30], which implements DFT with pseudopotentials and numerical basis sets of finite range. We used TroullierMartins pseudopotentials[31], and we applied local density approximation[32] for exchange-correlation potential. We tested various multiple-zeta basis sets to check dependence of the results on the basis. We chose a single-zeta basis with polarization as a fair compromise between accuracy and manageability for a total system size of 17 CNT unit cells, ie. $17 \times 40=680$ atoms in the unit cell. The ranges of the pseudoatomic orbitals (PAOs) were defined with an energy shift of $0.005 \mathrm{Ry}$.

We used a rectangular unit cell of dimensions: $32 \times 32 \times 42.1356 \AA^{3}$, with periodic boundary conditions along the transport direction $z$. The cell was large enough to avoid interactions between unit cells along $x, y$ directions. We defined the real space grid with a mesh cutoff of $200 \mathrm{Ry}$ and we used $5 k$ points along the CNT $z$ axis. The density matrix accuracy was set to $10^{-5}$. These parameters ensured the convergence of the results for the smooth pseudopotential employed.

We used a conjugate gradient method for relaxing the structure of the central region of the system (13 CNT periodicity units). The structures were assumed to be relaxed when the forces on the carbon atoms were smaller than $0.04 \mathrm{eV} / \AA$. Throughout the relaxations we kept fixed the boundary regions of our structures, i.e. those that represent parts of the perfect leads ( 2 CNT unit cells on each side of the scattering region).

We obtained the PCP of charge $+|e|(+2|e|)$ from a single $\mathrm{H}(\mathrm{He})$ atom, by reducing the size of the atom's basis function so that the energy of its atomic orbital exceeded the Fermi energy of the 
leads. As a result, the probe atom lost its electron(s) to the central part of the device and remained positively charged. The whole unit cell remained neutral throughout the computations, and the probe potential led to the formation of an image charge on the CNT surface near the probe position.

The transport computations were performed with the GOLLUM package[33] which uses the quasiparticle system Hamiltonian obtained from SIESTA to perform quantum transport computations within the framework of the Landauer-Büttiker formalism. GOLLUM does not need selfconsistent computations of the density matrix using Green's functions for the open system to calculate the transport coefficients. This is an important technical advantage in the present case, since in computing the conductance map for many PCP positions $\left(\sim 10^{3}\right.$ for this work) we needed each simulation to be as quick as possible and use the least possible memory resources.

We carried out calculations in four stages. First we computed the optimal structure of the infinite perfect CNT. The optimized CNT structure did not have any noticeable distortion from a perfect cylindrical symmetry, as expected. The resulting carbon-carbon distance was $1.431 \AA$, which is in good agreement with the experimental data and supports our choice of the computational parameters. Next, starting from the perfect CNT structure we introduced the point defect and computed the relaxed structure of the central region. In the third stage, we used the obtained relaxed structure of the previous stage as an input for the computations with the PCP for all the probe positions of the conductance map and relaxed the system again for each of them. Finally, we used the system Hamiltonians of SIESTA for the relaxed structures obtained in the previous stages as an input for the computations of the zero-voltage conductance using GOLLUM.

\section{Discussion of the results}

Point-like defects are known to cause the formation of quasibound states (QBS) in both the electronic structure and transport properties of CNTs[34, 35]. The electronic structure includes a narrow Lorentzian peak centered at an energy $E_{d}$, which is visible in the partial density of states (PDOS)[36] and the local density of states (LDOS)[35] for atoms in the neighborhood of the defect. The value of $E_{d}$ depends on the strength of the perturbation of the original CNT structure. For weak defects $E_{d}$ is close to the edge of the central two-band region. As the perturbation increases the QBS peak approaches the Fermi level.

The effect of the QBS in the conductance near $E_{F}$ consists in suppressing one of the conducting channels in a narrow energy interval corresponding to the QBS feature observed in the PDOS. As a result, the transmission is reduced from a value of 2.00, corresponding to the transmission of a perfect two-channel system, to a value of 1.00 at $E=E_{d}$. The effects of the vacancy and the 5775



Figure 2: (color online) Transmission function versus energy for a $(10,10)$ CNT with a single defect. (a) The CNT with the vacancy (circles) and the 5775 defect (diamonds) in absence of the probe. (b) CNT with the vacancy. (c) CNT with the 5775 defect. Solid lines: transmission in absence of the $\mathrm{PCP}$, broken lines: results for a PCP with $Q=+|e|$ located at the boundary of the scanning region, dashed-dotted lines: results for the PCP at the center of the scanning region.

defect on the transmission functions of our model system are shown in figure 2 for the energy range: $-1.5 \mathrm{eV}<E-E_{F}<1.5 \mathrm{eV}$. In the absence of the PCP the position of the QBS dip corresponding to 5775 defect agrees with the results of other authors $\left(E_{5775} \approx 0.45 \mathrm{eV}\right.$, see panel $\left.(\mathrm{a})\right)[37,35]$. The transmission dip of the QBS for the vacancy is located significantly closer to $E_{F}\left(E_{\mathrm{vac}} \approx 0.13 \mathrm{eV}\right)$. The latter value, as well as the overall energy dependence of the transmission, is in good agreement with the results of Rocha et al. [36].

In panels (b,c) of figure 2 we show the dependence of the transmission $T(E)$ near $E_{F}$ on the po- 
sition of the PCP probe of charge $Q=+|e|$ located near the defect. The net potential of the PCP and the image charge shifts the energy of the QBS near the Fermi level without a noticeable change of the shape of the dip. Although the center of the depression never reaches the Fermi level, the overlap of its shoulder with the band center grows with the probe approaching to the defect (see figure $2(a, b)$ ). In effect, the value of the transmission at $E_{F}$, and consequently the zero voltage conductance of the device: $\Gamma=\frac{2 e^{2}}{h} T\left(E_{F}\right)$, can be controlled by the position of the PCP.

By varying the position of the probe along a rectangular region parallel to the CNT axis and centered at the defect we obtained 2-dimensional maps of the transmission for the considered defects (see figure 3 for the 5775 defect and and figure 4 for the vacancy). The corners of the rectangular scanning (a)

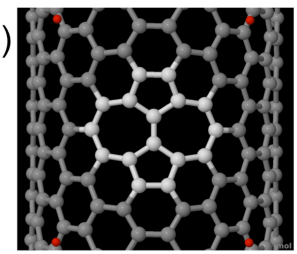

(b)



(c)



(d)



Figure 3: (color online) The CNT with the 5775 defect. (a) The relaxed atomic structure with highlighted atoms of the defect. (b) Local density of states (LDOS) computed at the energy corresponding to the dip of the quasibound state in the conductance. The isosurface value for LDOS plot is $0.002 \mathrm{eV}^{-1} \mathrm{Bohr}^{-3}$. The transmission map for the $Q=+|e|$ probe (c), and the $Q=+2|e|$ probe $(\mathrm{d})$.

region are indicated by the red dots in panels (a) of figures 3, 4, and the atoms forming the defect structure are highlighted. The panels (b) of the figures show the isosurfaces of the local density of states (LDOS) in absence of PCP. The LDOS function is computed for the energy regions corresponding to dips in the transmission function shown in figure 2(b,c) with solid lines. The limited extension of the LDOS contours confirm that the transmission dips can indeed be attributed to scattering by the


Figure 4: (color online) As in figure 3, but for the vacancy defect.

quasibound states.

The 2-dimensional maps of the transmission at the Fermi level $\left(T\left(E_{F}\right)\right)$ are presented in figures 3 and 4 , for the PCP charge of $Q=+|e|$ (panel (c)) and $Q=+2|e|$ (panel (d)). In the case of the weaker probe, the distance between the scanning region and the reference surface of the perfect CNT was set to $0.35 \mathrm{~nm}$, and for the stronger probe the distance was equal to $0.45 \mathrm{~nm}$. Note, that the blue (red) pixels correspond to the low (high) $T\left(E_{F}\right)$ values, and the gray pixels indicate the $T\left(E_{F}\right)$ values that are mean values of the $T\left(E_{F}\right)$ maximum and minimum $\left(T_{\text {mean }}\right)$ in the maps.

In the case of the 5775 defect and the PCP with $Q=+|e|$ charge, variations of $T\left(E_{F}\right)$ along the scanning region were less than $5 \%$. The minimal value of the transmission was reached for the probe position in the middle of the scan, at the rotated C$\mathrm{C}$ bond of the 5775 defect. For the stronger probe the variations of $T\left(E_{F}\right)$ were almost an order of magnitude bigger and reached about $38 \%$. However the colored regions in panels (c) for $Q=+|e|$, and (d) for $Q=+2|e|$ of figure 3 look similar to one another. In particular the gray oval contours of $T_{\text {mean }}$ have approximately similar sizes along the both directions.

The conductance map for the vacancy defect differs from the one obtained for the 5775 defect. The oval contour formed by pixels with $T\left(E_{F}\right)=T_{\text {mean }}$ values is clearly more rounded as compared to the former case. Also, for the vacancy defect the contour shape does not depend too much on the charge 
of the PCP. In the present case the transmission variations along the scanning region is about $20 \%$, for the PCP with $Q=+|e|$ charge, and they reach about $42 \%$, for the PCP with $Q=+2|e|$ charge.

In figure 5 we compare values of $T\left(E_{F}\right)$ for both defects and the PCP charges $Q=+|e|$ and $Q=$ $+2|e|$, for the PCP traveling along the line parallel to the CNT axis and going through the center of the scanning region. The broken lines in figure 5 show the results obtained for the weaker probe and linearly scaled to match the minimum and maximum values of $T\left(E_{F}\right)$ obtained for the stronger probe. In the case of the 5775 defect the good agreement of the scaled plot and the one for the stronger probe suggests that the shape of the dependence of $T\left(E_{F}\right)$ on the PCP position is universal one, ie. it is independent of the PCP charge and its distance from the CNT surface. The dependence on the PCP position for the 5775 defect exhibits an inflection point about $0.37 \mathrm{~nm}$ from the center. The latter value, which appears to be universal in the above mentioned sense, may be used to define an effective size of the defect along the $z$ direction for the purpose of a SPM measurement. For the vacancy

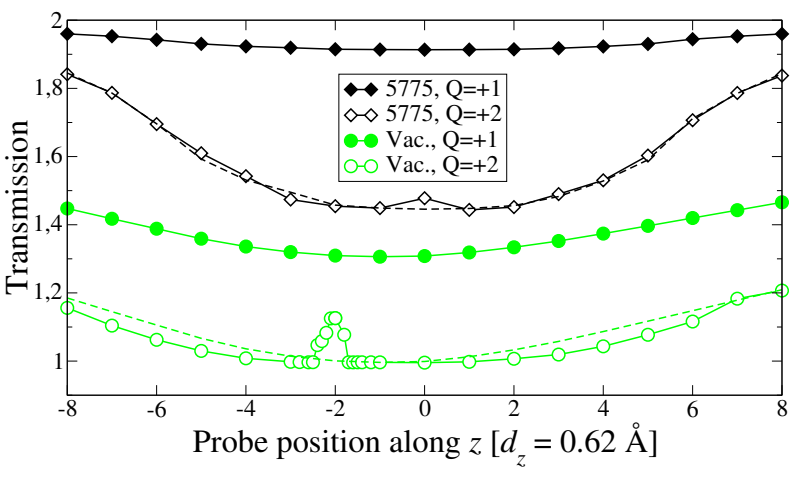

Figure 5: (color online) The transmission at $E=E_{F}$ for the CNT with the defect with respect to the position of the probe. The probe moves along the CNT axis through the center of the scanning region. Diamonds: 5775 defect, circles: vacancy. Full symbols are for $Q=+|e|$ probe, empty symbols for $Q=+2|e|$, broken lines are the corresponding results scaled with a linear transformation for $Q=+|e|$ probe.

defect the universality of the of dependence $T\left(E_{F}\right)$ on the probe position does not seem to hold so well as in the previous case. A possible reason may be that for the stronger probe this defect is close to a structural instability driven by the PCP potential. A particularly sensitive PCP location is near the carbon atom with an unsaturated bond. At this point the electron cloud filling the vacancy region, which is related to the lobe of LDOS shown in figure 4(b), is pushed out from the CNT surface by the strong electrostatic field of the probe. With the electron cloud diverted now in the direction of the probe, the CNT surface is partly discharged and a substantial reconstruction of the vacancy region and its neighborhood takes place. The probe induced reconstruction of the defect region manifests itself also as a sharp peak in the $T\left(E_{F}\right)$ dependence on the PCP position at $Q_{Z}=-2$ pixel and is visible as a lighter pixel in the center of the map in figure 4.

The vacancy defect considered above includes a single unsaturated bond and for this reason is very reactive[9] and may be stable in carbon-based materials only in ultrahigh vacuum conditions[22]. In presence of ambient atmosphere the vacancy binds atoms of gases and transforms itself to a more stable passivated form. The effect of gas adsorption at the vacancy site on the conductance of a CNT can be applied in gas sensors and with this motivation it was analyzed previously[38]. Here we study the influence of hydrogen adsorption at the vacancy site on the conductance, in the presence of the charged probe. We consider two representative cases of $\mathrm{H}$-passivated vacancies: a single $\mathrm{H}$ atom saturating one dangling bond and three $\mathrm{H}$ atoms saturating three bonds. The spatial and electronic structure of these defects was studied earlier by other authors[39, 40, 22], and the relaxed structures of the defects obtained here are in good agreement with these works (see figure 6 ). The bare vacancy can be viewed as a deep donor level in the vicinity of $E_{F}$. Upon the adsorption of a single hydrogen atom at the dangling bond of the vacancy it transforms into an acceptor-like defect (figure 6a,b). This is evident from a shift of the dip of the corresponding quasibound state below $E_{F}$. Adsorption of three $\mathrm{H}$ atoms at the dangling bonds of the unreconstructed vacancy (figure 6c) results in a quasibound state located just below $E_{F}$, and strongly reducing the transmission at $E=E_{F}$.

The transmission maps obtained for the CNT with the passivated vacancies show that the transmission attains its maximum for the probe position near the defect center. This is in contrast with the behaviour of the bare vacancy, where the transmission decreases with the PCP approaching the defect (cf. figures: 4c,d and $6 \mathrm{~d}, \mathrm{e}$ ). This difference is due to the different location of the transmission dip with respect to the Fermi level for the bare and the 
(a)



(b)



(c)



(d)



(e)



Figure 6: (color online) The transmission function for the $(10,10)$ CNT with the vacancy passivated with one or three hydrogen atoms. (a) Transmission vs. energy in absence of the PCP probe for the bare vacancy (full circles), singly $\mathrm{H}$-passivated vacancy (squares) and triply $\mathrm{H}$-passivated vacancy (empty circles) (b) Relaxed structure of CNT near the vacancy passivated with a single $\mathrm{H}$-atom. (c) The same as (b) but for the vacancy passivated with three H-atoms. (d,e) Transmission maps for the $Q=+|e|$ probe positioned about $4.5 \AA$ above the CNT surface for the H-passivated vacancies shown in $(\mathrm{b}, \mathrm{c})$, respectively.

passivated vacancies. In general the potential of the PCP probe decreases the energy of the QBS located near $E_{F}$. While for the bare vacancy it results in shifting the transmission dip near $E_{F}$ and reducing the transmission, for the passivated vacancies it leads to removing the transmission dip from the vicinity of $E_{F}$ and a concomitant increase of the transmission.

To rationalize our numerical results we use a tight-binding $\pi$-electron model of a metallic CNT with a point defect of strength $E_{D}$. The analytical formula for the conductance obtained exactly within a framework of the model[41, 42] reads:

$$
\Gamma / \Gamma_{0}=1+\frac{1}{1+\left(E_{D} / C t\right)^{2}},
$$

where $C=N \sqrt{3}$ for $(N, N)$ armchair CNT, $C=N$ for a metallic $(N, 0)$ zigzag CNT, $t$ denotes the hop- ping parameter and $\Gamma_{0}$ is the conductance quantum. We assume that the influence of the electrostatic potential due to the PCP and the image charge on the defect energy can be to approximately represented with the following two-parameter shift function:

$$
E_{D} \rightarrow E_{D}(z)=E_{D}+E_{0} /\left[1+(\gamma z)^{2}\right]^{3 / 2}
$$

The parameters $E_{0}, \gamma$ in equation (2) describe the effect of the charge of the PCP and its distance from the CNT axis, and the decay of the image charge potential along the CNT, respectively. The exponent $3 / 2$ in the above formula takes into account the fact that at large distances of the PCP from the defect site the resulting potential of the PCP and the image charge is effectively seen as that of a dipole.

The parameter $E_{D}$ can be fixed by comparing the value of $\Gamma$ computed in absence of the PCP with the corresponding quantity computed from equation (1). In this way we obtain: $E_{D} / C|t|=0.15$ for 5775 defect, $E_{D} / C|t|=0.55$ for the bare vacancy, $E_{D} / C|t|=-0.8$ for the single-passivated vacancy and $E_{D} / C|t|=-4.0$ for the triple passivated vacancy. In a similar way the parameters $E_{0}, \gamma$ can be found. Their values depend on the electron charge distribution around each defect and for this reason they are also defect dependent. We found that the values of $E_{0}$ are all positive, which means that the potential contribution from the negative image charge dominates $E_{0}$. As a result, for the probe approaching the defect the transmission increases for negative $E_{D}$, whereas it decreases in the case of positive $E_{D}$. Moreover, the computed values of $E_{0}$ are of the same order of magnitude as $E_{D}$. The latter finding follows from the fact that stronger defects perturb more the image charge near the defect. Finally, the value of $\gamma$ that reproduced our DFT results was found to be of order of $0.1 \AA^{-1}$.

On the basis of the above arguments, used to explain the value of $E_{0}$, we can now understand the stronger variation of the transmission along the scanning region for stronger defects. We can also qualitatively explain the changes of the variation with the value and sign of the probe charge or the dependence on the distance of the probe from the CNT surface as well as the minimum (maximum) of the transmission at the defect site for donor (acceptor) type defects. However the prediction of the defect specific contour in the transmission map is beyond the scope of the $\pi$-electron model analysis 
and for this purpose our first principles computations are indispensable.

\section{Summary and conclusions}

By using first-principles simulations based on DFT we computed different transmission maps, obtained by varying the position of the PCP along the surface of the armchair $(10,10)$ CNT including selected defects. The maps were obtained for two values of the charge of the probe $(Q=+2|e|$ and $Q=+|e|)$. Two intrinsic defects were considered: a 5775 defect and a bare vacancy, which were earlier experimentally confirmed to exist in CNTs[19] and graphitic layers[22]. We also studied the effect of hydrogen passivation of the vacancy on the system properties.

Each of the studied defects leads to a quasibound state in the central two-band region of the CNT and the corresponding dips in the electronic transmission. Depending on the location of the nearest transmission dip with respect to the Fermi level the defects can be viewed as donor-like (5775 defect and the bare vacancy) or acceptor like (the $\mathrm{H}$ - passivated vacancies). We showed that the positively charged probe located near the center of each defect in each case shifts downwards the nearest transmission dips. As a result, the positive PCP reduces the transmission for the donor-like defects and increases the transmission for the acceptor-like defects.

We noticed clear differences between the maps obtained for different intrinsic defects: the 5775 defect and the bare vacancy. Moreover the maps obtained for the same defect and different magnitudes of the PCP potentials and distances from the CNT surface appear similar if we apply a simple linear scaling. Hence we conclude that the pattern visible in the conductance map is a universal property of the defect and should allow to identify the defect's type in experiments performed with scanning probe microscopy methods.

A specific feature of the conductance map obtained using scanning gate microscopy is that it reflects the spatial dependence of the local density of states related to the defect-induced QBS, rather than the positions of particular atoms forming the structure of the defect $[44,45]$. The spatial size of the QBS seen in our computations sets a length scale of around $1 \mathrm{~nm}$ as working distance of the probe from the CNT in the SPM measurements aimed to identify the defect. For distances much larger than the QBS size the probe potential would be seen as an almost uniform field in the vicinity of the defect. As a result the probe would play the role of a usual gate and the specific contour of the defect in the conductance map would be obliterated.

A typical lateral resolution of scanning gate microscopy is of the order of tens on nanometers $[2,10]$ and it is limited because of the long range of the Coulomb potential and the unknown precise shape and size of an SGM tip[45]. For this reason SGM investigations, while being able to locate the individual defects, do not possess sufficient resolution to unambiguously identify them. By simultaneously collecting the source-drain voltage and back-gate voltage and creating defects under chemically controllable conditions[46] the identity of the defect could be established. The results of our work suggest an alternative approach to the latter elaborate method. Instead of using a conducting tip, as is commonly done in the SGM in order to control the tip's potential, we propose using a possibly large insulating tip functionalized with a charged atom or cluster of atoms. In such case the probe electrostatic potential could be more precisely determined and it would not much depend on details of a tip's shape. Note that in order to get a usable scan of the defected region a precise knowledge of the charge location on the tip would not be necessary, however the stability of its position would be important. Such a procedure seems feasible in the light of experiments done with AFM tips where charged particles were picked up from the supporting surface and were shown to modify the scanned image[43].

Since we restricted ourselves to scattering by a single defect, which could also apply to systems with a very small density of defects, the results of the present work cannot directly describe CNTs with large number of defects, as those created by high radiation doses[18]. In such cases interference effects due to scattering from different defects would introduce large conductance oscillations $[47,48]$ that may spoil the map. However for CNTs with a substantial number of defects, our results may still be valid as long as the average distance between the defects is not much smaller than the mean-free-path for inelastic scattering of electrons.

In the computations we relaxed the CNT structure for each position of the defect. For most of the probe positions the impact of the relaxation, although clearly noticeable, does not change much the characteristic patterns seen in the transmission 
maps. However, for the vacancy defect the stronger probe was found to lead to a local structural instability. This effect was due to a charge redistribution around the unsaturated bond of one of the carbon atoms. This finding may suggest a possible explanation of the instabilities noticed for unspecified defects in experimental works[20, 46].

Finally, the results of our work indicate roads for possible applications of local defects in metallic CNTs. The substantial sensitivity of the defected region to the electric field of PCP could be used for local charge mapping in surfaces of solids or in nanoelectronic devices. Alternatively, the same effect may be employed to produce local potential meters, working with a subnanometer precision.

\section{Acknowledgments}

This work has been supported by the National Science Centre under the contract DEC2012/07/B/ST3/03412. We acknowledge that the results of this research have been achieved using the PRACE-3IP project (FP7 RI-312763) resource JUROPA based in Germany at Julich. We also thank the Spanish MICINN for the Grant No. FIS201234858 (V.M.G.S. and J.F.) and the Ramón y Cajal Fellowship No. RYC-2010-06053 (V.M.G.S.). The authors thanks Nicolás Agraït and Maciej Wiesner for useful discussions of the experimental details.

[1] J.-C. Charlier, Acc. Chem. Res. 35 (2002) 1063-1069.

[2] P. G. Collins, Defects and disorder in carbon nanotubes, in: A. V. Narlikar, \& Y. Y. Fu (Eds.), Oxford Handbook of Nanoscience and Technology: Frontiers and Advances. Oxford Univ. Press, Oxford, 2010.

[3] A. V. Krasheninnikov and K. Nordlund, Appl. Phys. Rev. 107 (2010) 071301-70.

[4] C. T. White and T. N. Todorov, Nature 393 (1998) 240242 .

[5] T. Ando, J. Phys. Soc. Jpn. 74 (2005) 777-817.

[6] J.-C. Charlier, X. Blase, S. Roche, Rev. Mod. Phys. 79 (2007) 677-732.

[7] V. Sgobba and D. M. Guldi, Chem. Soc. Rev. 38 (2009) 165-184.

[8] M. Burghard, H. Klauk, and K. Kern, Adv. Mater. 21 (2009) 2586-2600.

[9] E. O. Pentsak, A. S. Kashin, M. V. Polynski, K. O. Kvashnina, P. Glatzel and V. P. Ananikov, Chem. Sci. 6 (2015) 3273-3636.

[10] S. J. Tans and C. Dekker, Nature (London) 404 (2000) 834-834.

[11] A. Bachtold, M. S. Fuhrer, S. Plyasunov, M. Forero, E. H. Anderson, A. Zettl and P. L. McEuen, Phys. Rev. Lett. 84 (2000) 6082-6085.

[12] M. Freitag, M. Radosavljevic, W. Clauss, and A. T. Johnson, Phys. Rev. B 62 (2000) R2307-R2310.

[13] M. Bockrath, W. Liang, D. Bozovic, J. H. Hafner, C. M. Lieber, M. Tinkham, H. Park, Science 291 (2001) 283-285.
[14] M. Freitag, A. T. Johnson, S. V. Kalinin, and D. A. Bonnell, Phys. Rev. Lett. 89 (2002) 216801-4.

[15] J.Y. Park, Appl. Phys. Lett. 90 (2007) 023112-3.

[16] A. J. Stone and D. J. Wales, Chem. Phys. Lett. 128 (1986) 501-503.

[17] M. B. Nardelli, B. I. Yakobson, and J. Bernholc, Phys. Rev. B 57 (1998) R4277-R4280.

[18] C. Gomez-Navarro, P. J. de Pablo, J. Gomez-Herrero, B. Biel, A. Rubio, and F. Flores, Nature Materials 4 (2005) 534-539.

[19] K. Suenaga, H. Wakabayashi, M. Koshino, Y. Sato, K. Urita and S. Iijima, Nature Nanotech. 2 (2007) 358-360.

[20] A. W. Bushmaker, C. C. Chang, V. V. Deshpande, M. R. Amer, M. W. Bockrath, S. B. Cronin, IEEE Trans. on Nanotech. 10 (2011) 582-586.

[21] B. M. Maciejewska, M. Jasiurkowska-Delaporte, A. I. Vasylenko, K. K. Kozioł and S. Jurga, RSC Adv. 4 (2014) 28826-28831.

[22] M. Ziatdinov, S. Fujii, K. Kusakabe, M. Kiguchi, T. Mori, and T. Enoki, Phys. Rev. B 89 (2014) 155405-15.

[23] L.-G. Tien, C.-H. Tsai, F.- Y. Li, and M. H. Lee Phys. Rev. 72 (2005) 245417-6.

[24] Y.W. Son, J. Ihm, M. L. Cohen, S. G. Louie, and H. J. Choi, Phys. Rev. Lett. 95 (2005) 216602-4.

[25] Y. W. Son, M. L. Cohen, and S. G. Louie, Nano Lett. 7 (2007) 3518-3522.

[26] Y. P. Luo, L. G. Tien, C. H. Tsai, M. H. Lee, and F. Y. Li, Chin. Phys. B 20 (2011) 017302-5.

[27] V. Meunier, S. V. Kalinin, J. Shin, A. P. Baddorf, and R. J. Harrison, Phys. Rev. Lett. 93 (2004) 246801-4.

[28] S. Iijima and T. Ichihashi, Nature 363 (1993) 603-605.

[29] The figures with structures of CNTs were made using Jmol: an open-source Java viewer for chemical structures in 3D. http://www.jmol.org/.

[30] P. Ordejon, E. Artacho and J. M. Soler, Phys. Rev. B 53 (1996) R10441-R10444; J. M. Soler, E. Artacho, J. D. Gale, A. García, J. Junquera, P. Ordejón, and D. Sánchez-Portal, J. Phys. Condens. Matter 14 (2002) 2745-2779.

[31] N. Troullier and J. L. Martins, Phys. Rev. B 43 (1991) 1993-2006.

[32] D. M. Ceperley and B. J. Alder, Phys. Rev. Lett. 45 (1980) 566-569.

[33] J. Ferrer, C. J. Lambert, V. M. Garcia-Suarez, D. Z. Manrique, D. Visontai, L. Oroszlany, R. RodriguezFerradas, I. Grace, S. W. D. Bailey, K. Gillemot, H. Sadeghi and L. A. Algharagholy, New J. Phys. 16 (2014) 093029-66.

[34] T. Kostyrko, M. Bartkowiak, and G. D. Mahan, Phys. Rev. B 59 (1999) 3241-3249.

[35] H. J. Choi, J. Ihm, S. G. Louie, and M. L. Cohen, Phys. Rev. Lett. 84 (2000) 2917-2920.

[36] A. R. Rocha, J. E. Padilha, A. Fazzio, and A. J. R. da Silva, Phys. Rev. B 77 (2008) 153406-4.

[37] H. J. Choi and J. Ihm, Phys. Rev. B 59 (1999) 22672275.

[38] Z. Zanolli and J.-C. Charlier, Phys. Rev. B 80 (2009) $155447-6$.

[39] Y. C. Ma, P. O. Lehtinen, A. S. Foster, and R. M. Nieminen, Phys. Rev. B 72 (2005) 085451-6.

[40] S.-H. Jhi, Carbon 45 (2007) 2031-2036.

[41] H. J. Choi and J. Ihm, Solid State Commun. 111 (1999) 385-390.

[42] T. Kostyrko, Phys. Rev. B 62 (2000) 2458-2465.

[43] C. Barth, T. Hynninen, M. Bieletzki, C. R. Henry, A. 
S. Foster, F. Esch and U. Heiz, New J. Phys 12 (2010) 093024-14.

[44] F. Martins, B. Hackens, M. G. Pala, T. Ouisse, H. Sellier, X. Wallart, S. Bollaert, A. Cappy, J. Chevrier, V. Bayot, and S. Huant, Phys. Rev. Lett. 99 (2007) 1368074.

[45] H. Sellier, B. Hackens, M. G. Pala, F. Martins, S. Baltazar, X. Wallart, L. Desplanque, V. Bayot, and S. Huant, Semicond. Sci. Technol. 26 (2011) 064008-10.

[46] S. R. Hunt, E. J. Fuller, B. L. Corso, and P. G. Collins, Phys. Rev. B 85 (2012) 235418-11.

[47] M. P. Anantram and T. R. Govindan, Phys. Rev. B 58 (1998) 4882-4887.

[48] T. Kostyrko, M. Bartkowiak, and G. D. Mahan, Phys. Rev. B 60 (1999) 10735-10738. 